\title{
Heurística y metaheurísticas en la recolección de residuos tecnológicos
}

\section{Heuristic and metaheuristics in e-waste collection}

\author{
Miguel López, Andrea Villagra, Daniel Pandolfi \\ milopez@uaco.unpa.edu.ar,avillagra@uaco.unpa.edu.ar,dpandolfi@uaco.unpa.edu.ar \\ Laboratorio de Tecnologías Emergentes (LabTEm) \\ Instituto de Tecnología Aplicada (ITA) - Unidad Académica Caleta Olivia \\ Universidad Nacional de la Patagonia Austral - Santa Cruz - Argentina
}

Recibido: 01/09/2021. Aceptado: 16/12/2021

\section{RESUMEN}

Los residuos tecnológicos generan contaminación ambiental y la recolección de residuos produce emisión de gases de efecto invernadero generado principalmente por vehículos de combustibles fósiles utilizados en las rutas de recolección. Por esta razón, es importante realizar una gestión eficiente de los residuos tecnológicos que generan contaminación y optimizar a través de diversas técnicas las rutas de recolección.

En este trabajo se aplican a instancias reales de las ciudades de Caleta Olivia y Comodoro Rivadavia tres técnicas: la heurística del algoritmo de ahorros y dos metaheurísticas Búsqueda tabú y Optimización basada en colonia de hormigas para encontrar la que mejor optimice el recorrido. Sobre las instancias utilizadas se han obtenido los mejores resultados en cuanto a los kilómetros recorridos aplicando al Búsqueda Tabú.

Palabras clave: Problema de Ruteo de Vehículos; Heurística; Metaheurísticas; Optimización; Residuos Tecnológicos.

\begin{abstract}
E-waste generates environmental pollution and the collection of waste produces greenhouse gas emissions, mainly generated by fossil fuel vehicles used in the collection routes. For this reason, it is important to efficiently manage the technological waste that generates pollution and to optimise the collection routes through various techniques.

In this work, three techniques are applied to real instances in the cities of Caleta Olivia and Comodoro Rivadavia: the heuristic of the savings algorithm and two metaheuristics, Tabu Search and Ant Colony Optimisation, to find the one that best optimises the route. On the instances used, the best results in terms of kilometres travelled have been obtained by applying Tabu Search.
\end{abstract}

Keywords: Vehicle Routing Problem; Heuristics; Metaheuristics; Optimisation; Technological Waste. 


\section{INTRODUCCIÓN}

Los grandes volúmenes de residuos que se generan actualmente en las zonas urbanas crean un problema en la definición de las rutas y localización de la flota vehicular de recolección. En términos computacionales, toda esta información se convierte en grandes volúmenes de datos que se deben almacenar para la toma decisiones, teniendo en cuenta restricciones como: las distancias a recorrer, el número de vehículos, su capacidad, factores ambientales, tráfico, semaforización, horas pico, zonas urbanas y contaminación acústica. Todo esto conlleva a un aumento computacional significativo en costo y complejidad algorítmica (Balari, 2017).

Algunos tipos particulares de residuos (como por ejemplo residuos tecnológicos, residuos hospitalarios, entre otros) deben ser recolectados y transportados en el menor tiempo posible, debido a su impacto pasivo en la salud de las personas. En particular, durante la pandemia mundial de COVID-19, se destaca la importancia y la necesidad de dar prioridad a la eliminación de los residuos médicos.

Los equipos eléctricos y electrónicos (EEE, Electrical and Electronic Equipment) se convierten en uno de los grupos más importantes de residuos que contienen materiales fáciles de reciclar. Los desechos electrónicos son conocidos como RAEE (en español, Residuos de Aparatos Eléctricos y Electrónicos) o bien e-waste o WEEE (en inglés, Waste Electrical and Electronic Equipment). La tasa de $e$-waste está creciendo a un ritmo alarmante, especialmente en los países desarrollados.

En América Latina solamente unos pocos países tienen proyectos de ley específicos sobre la gestión de los desechos electrónicos. En la mayoría de los casos, la gestión de los residuos electrónicos está regulada en la legislación general de residuos peligrosos. Actualmente se debaten políticas públicas específicas o directrices técnicas, que aún deben pasar por el proceso legislativo o ser implementadas.

En Argentina, algunas provincias tienen legislación específica RAEE, por ejemplo, la provincia de Buenos Aires desde 2011. Un marco legislativo nacional para el $e$-waste ha sido discutido en el parlamento durante algunos años. Los principios generales de gestión de residuos del país están en línea con los principios de la Convención de Basilea (1990). Además, la propia Constitución garantiza el derecho a un medio ambiente sano, equilibrado y adecuado para el desarrollo humano, e insta a las autoridades a aplicar esta disposición y aumentar la conciencia y la educación. Recientemente, se han puesto en marcha diversas iniciativas que significan un avance en materia de $e$-waste.

Las actividades en la recolección de residuos se pueden agrupar en cinco etapas correspondientes al ciclo de vida de los residuos: generación, recolección y transporte, transformación, tratamiento y eliminación final (Nesmachnow et al. 2018, Ghiani et al. 2012). El costo de la recolección y transporte de residuos representa entre el 60 y el $80 \%$ de los costes totales del Sistema de Gestión de Residuos (SGR), que es el factor crítico en el gasto fiscal de los SGR, y por esta razón las mejoras en este campo representan un importante papel en el ahorro del gasto municipal (Babaee Tirkolaee et al. 2019)

Dada la importancia de la recolección y transporte este trabajo se centra en esta etapa. La recolección de residuos se refiere al uso de vehículos de recolección de residuos para cargar los residuos desde los puntos de recolección. El transporte de residuos se refiere a la actividad de llevar los residuos recolectados al centro de eliminación Se han propuesto diferentes 
técnicas para su resolución tanto exactas, heurísticas y metaheurísticas (Laporte et al. 2000, Asih et al. 2017). El término heurística proviene del vocablo griego heuriskein que podría traducirse como encontrar, hallar o descubrir. Actualmente, existen bastantes definiciones para el término heurística. Una de las más claras e intuitivas es la presentada por Zankis y Evans (1981): "Procedimientos simples, a menudo basados en el sentido común, que se supone que obtendrán una buena solución (no necesariamente óptima) a problemas difíciles de un modo sencillo y rápido".

Los métodos heurísticos tienen su principal limitación en su incapacidad para escapar de óptimos locales. Esto se debe, fundamentalmente, a que estos algoritmos no utilizan ningún mecanismo que les permita proseguir la búsqueda del óptimo en caso de quedar atrapados en un óptimo local. Para resolver este problema se introducen otros algoritmos más inteligentes (denominados metaheurísticas, Glover F. 1986) que evitan, en la medida de lo posible este problema. Este tipo de algoritmos son procedimientos de alto nivel que guían a métodos heurísticos conocidos, evitando que éstos queden atrapados en óptimos locales.

El objetivo de este trabajo es estudiar el problema de ruteo de vehículos con capacidad limitada aplicado a la recolección de residuos tecnológicos para determinar que procedimiento heurístico y metaheurísticos resulta más eficiente para resolver escenarios reales de las ciudades de Caleta Olivia (Santa Cruz) y Comodoro Rivadavia (Chubut). Se implementa el algoritmo de ahorros (al que denominaremos AA) propuesto por Clarke y Wright (1964) muy utilizado para problemas de ruteo de vehículo y dos metaheurísticas: Búsqueda Tabú (en inglés Tabu Search - TS) y Optimización basada en Colonia de Hormigas (en inglés Ant Colony Optimization -ACO) esta última también muy aplicada en este tipo de problemas.

El artículo está organizado de la siguiente manera: la Sección 1 presenta una revisión de la literatura, la Sección 2 describe el problema de ruteo de vehículos. La Sección 3 muestra la descripción de los algoritmos utilizados para resolver el problema, la Sección 4 describe las instancias, los experimentos realizados y resultados obtenidos. Finalmente, la Sección 5 presenta las conclusiones.

\section{REVISION DE LA LITERATURA}

El problema de recolección de residuos ha sido abordado por numerosos autores con diversas técnicas, tanto por medio de métodos heurísticos como exactos. En particular se encuentran artículos dedicados a la recolección de residuos sólidos urbanos y en nuestro país (Argentina) se encuentran trabajos aplicados a instancias reales como en Bonomo et al. (2009) donde implementan modelos de programación matemática para la recolección de residuos en la zona sur de la Ciudad de Buenos Aires. Además, trabajos de optimización de rutas y desgaste de los vehículos en Bonomo et al. (2012) y Bertero, F. (2015), optimización de recursos en Bianchetti, M. (2015) y se proponen modelos de ruteo de vehículos con balanceo de distancias entre las distintas rutas para diseñar los recorridos de los recolectores informales de residuos reciclables (Cavallin et al. 2014).

En cuanto a la recolección de residuos tecnológicos en países de Europa y Asia existen varios métodos, entre ellos recolección en puntos fijos, recolección de residuos móvil como de reciclaje en la vereda o en puntos móviles situados en zonas frecuentadas, o bajo demanda cuando se solicita la recolección de RAEE (Gu et al., 2019, Nowakowski et al. 2018). La tarea de recolección por demanda requiere el uso de algoritmos de inteligencia artificial para la optimización de las rutas de los vehículos y el número de éstos, ya que se trata de un problema típico de ruteo de vehículos con ventanas de tiempo (VRPTW) (Toth y Vigo, 2014). 
Las restricciones adicionales que se producen en la práctica empresarial (por ejemplo, capacidad máxima de carga útil del vehículo, capacidad de carga útil, aceptación de diferentes tipos de equipos por parte de los vehículos, heterogeneidad de los RAEE y de la flota, posible necesidad de descargar los residuos y continuar la recolección de residuos) obligan a ampliar el modelo clásico del Problema de ruteo de vehículo. Los problemas de carga de contenedores y los problemas de encaminamiento y carga de vehículos se analizaron un estudio de Nowakowski et al. (2017). Mar-Ortiz et al. (2013) analizan los problemas de enrutamiento de vehículos con cargas divididas y ventanas de fecha para los RAEE utilizando el procedimiento de búsqueda adaptativa aleatoria codiciosa.

Boussaïd et al. (2013), realizaron un estudio de un grupo de metaheurísticas de optimización. En particular, detallamos aquella inspiradas en las metáforas del comportamiento de enjambre en la naturaleza, este enfoque es conocido como Inteligencia Colectiva, (SI, Swarm Intelligent). Ejemplos de SI son Optimización de Colonias de Hormigas (ACO, Ant Colony Optimization), la Optimización de Cúmulo de Partículas (PSO, Particle Swarm Optimization), la Optimización de Forrajeo Bacteriano (BFOA, Bacterial Foraging Optimization Algorithm), Optimización de Colonias de Abejas (BCO, Bee Colony Optimization), Sistemas Inmunes Artificiales (AIS, Artificial Immune Systems) y Optimización Basada en Biogeografía (BBO, Biogeography-Based Optimization). Los algoritmos basados en SI se han aplicado con éxito a la resolución de problemas de ruteo de vehículos (en inglés, Vehicule Routing Problem VRP) y sus variantes. Una de las características más importantes de estos métodos se ve en su capacidad para adaptarse a la instancia y la consistencia resultante observada al aplicar cambios leves en el modelo y los parámetros del problema. Por lo tanto, es común adaptar enfoques a diferentes contextos en los problemas del mundo real, con relativamente pocos cambios mientras se adaptan perfectamente a las características encontradas en los problemas de planificación de rutas.

Yi et al. (2019), aplican para este problema el algoritmo de Harmoby Search (HS) y muestran que no requiere valores iniciales para las variables de decisión y, en comparación con algunas metaheurísticas populares, impone menos requisitos matemáticos y puede adaptarse fácilmente a diferentes problemas (Lee y Geem, 2005).

Por otra parte, Nowakowaski et al. (2020) proponen un algoritmo con inteligencia artificial que obtiene resultados muy promisorios. Un estudio reciente de Sun et al. (2020) analizan diferentes variantes de la recolección de RAEE como modelos de negocio con posibles oportunidades para las empresas de reciclaje. Esto indica el creciente interés del posible uso de las Tecnologías de la Información y la comunicación, Internet de las cosas y las plataformas basadas en la web para una amplia aplicación en la sociedad. La adquisición de datos sobre el tipo, número y volumen de residuos, incluida la ubicación del punto de recolección y las ventanas de tiempo de acceso, crean nuevas oportunidades y desafíos para las empresas de recolección de residuos.

A pesar de la adaptación de muchas metaheurísticas diferentes para resolver problemas derivados del VRP, asumimos que el problema de planificación de la recolección de RAEE para las instancias reales de las ciudades de Caleta Olivia y Comodoro Rivadavia puede ser resuelto por metaheurísticas de trayectoria permitiendo la obtención de soluciones rápidas y optimas. 


\section{DESCRIPCION DEL PROBLEMA}

Las distintas estrategias de recolección de residuos pueden asociarse al conocido problema de enrutamiento o ruteo de vehículos. Este problema fue planteado por primera vez por Dantzig y Ramser en 1959, cuando estos autores realizan una formulación del problema para una aplicación de distribución de combustible y lo definen como: "la determinación de la ruta óptima para una flota de vehículos que parten de uno o más depósitos para satisfacer la demanda de varios clientes dispersados geográficamente" (Dantzig y Ramser, 1959).

El VRP es uno de los problemas más conocidos y desafiantes en la programación lineal entera y es parte de los problemas llamados NP-Hard (Lenstra y Kan, 1981), que son los problemas que no se pueden resolver en un tiempo polinomial. El tiempo y esfuerzo computacional requerido para resolver este problema aumenta exponencialmente respecto al tamaño del problema. Por esta razón se han ideado algoritmos que no garantizan optimalidad, pero que logran entregar buenas soluciones a estos problemas difíciles de resolver como lo son las heurísticas y metaheurísticas.

Existen diferentes variantes del VRP, que incluyen restricciones adicionales y la incorporación de múltiples variables. Algunas restricciones más importantes son: (a) cada vehículo tiene capacidad limitada (en inglés, Capacited Vechicle Routing Problem - CVRP), (b) todos los clientes deben ser atendidos dentro de una cierta ventana de tiempo (en inglés, Vehicle Routing Problem with Time Windows - VRPTW), (c) el proveedor utiliza muchos depósitos para suministrar a los clientes (en inglés, Multiple Depot Vehicle Routing Problem MDVRP) (d) los clientes pueden devolver algunos productos (en inglés, Vehicle Routing Problem with Pick-up and Delivering -VRPPD) (e) los clientes pueden recibir servicio por diferentes vehículos (en inglés, Split Delivery Vehicle Routing Problem - SDVRP) (f) algunas variables del problema con consideradas aleatorias y varían en el tiempo como por ejemplo el número de clientes, sus demandas, el tiempo de servicio o el tiempo de viaje (VRP estocástico, en inglés, Stochastic Vehicle Routing Problem - SVRP) (g) las entregas se pueden realizar en algunos días (VRP periódico, en inglés Periodic Vehicle Routing Problem - PVRP).

En la actualidad el problema de recolección o distribución de productos a partir de un depósito original (punto de origen) y una cantidad de clientes con una demanda por atender, juega un papel importante en empresas logísticas ya que deben planificar inteligentemente las rutas de manera tal que signifiquen considerables ahorros en costos tales como: el consumo de combustible, horas hombre, entre otros; que ayudarán a una mejor rentabilidad para los negocios hoy en día. En relación con una creciente sensibilidad ambiental los problemas de enrutamiento de vehículos presentan una extensa disponibilidad bibliográfica de los problemas de enrutamiento verde de vehículos (GVRP). Los diferentes tipos de problemas de enrutamiento de VRP en logística inversa, describen perfectamente la complejidad en los casos del mundo real.

En este artículo trabajamos la variante de VRP con capacidad limitada (CVRP), en el que cada vehículo tiene una capacidad uniforme de un único artículo. Definimos el CVRP sobre un grafo no dirigido $G=(V, E)$ donde $V=\left\{v_{0}, v_{1}, . ., v_{n}\right\}$ es un conjunto de vértices y $E=\left\{\left(v_{i}\right.\right.$, $\left.\left.v_{j}\right) / v_{i}, v_{j} \in V, i<j\right\}$ es un conjunto de ejes.

El vértice $v_{0}$ es el depósito, y es desde donde $m$ vehículos de capacidad $Q$ deben abastecer a todas las ciudades o clientes, representados por un conjunto de $n$ vértices $\left\{\mathrm{v}_{l}, \ldots, v_{n}\right\}$. 
Definimos en $E$ una matriz $C=\left(c_{i j}\right)$ de costo, distancia o tiempo de viaje no negativos entre los clientes $v_{i}$ y $v_{j}$. Cada cliente $v_{i}$ tiene una demanda no negativa de artículos $q_{i}$ y tiempos de entrega $\delta_{i}$ (tiempo necesario para descargar todos los artículos). Siendo $v_{l}, . ., v_{m}$ una partición de $V$, una ruta $R_{i}$ es una permutación de los clientes en $V_{i}$ especificando el orden en el que se visitan, comenzando y terminando en el depósito $v_{0}$. El costo de una ruta dada $R_{i}=$ $\left\{v_{0}, v_{1}, . ., v_{k+1}\right\}$, donde $v_{j} \in V$ y $v_{0}=v_{k+1}=0$ ( 0 indican el depósito), viene dada por la ecuación (1):

$$
\operatorname{Cost}(R i)=\sum_{j=0}^{k} c_{i, j+1}+\sum_{j=0}^{k} \delta_{j}
$$

y el costo de la solución al problema $(S)$ es:

$$
\operatorname{FCVRP}(S)=\sum_{i=1}^{m} \operatorname{Cost}\left(R_{i}\right)
$$

El CVRP consiste en determinar un conjunto de $m$ rutas (i) de costo total mínimo - como especifica la ecuación (2); (ii) empezando y terminando en el depósito $v_{0}$; de forma que (iii) cada cliente es visitado una sola vez por un sólo vehículo, sujeto a las restricciones (iv) de que la demanda total de cualquier ruta no exceda $Q\left(\sum_{v_{j} \in R_{i}} q_{j} \leq Q\right)$; y (v) la duración total de cualquier ruta no supera el límite preseleccionado $D\left(\operatorname{Cost}\left(R_{i} \leq D\right)\right.$. Todos los vehículos tienen la misma capacidad y transportan el mismo tipo de artículo. El número de vehículos puede ser un valor de entrada o una variable de decisión. En este estudio, la longitud de las rutas se minimiza independientemente del número de vehículos utilizados.

\section{DESCRIPCION DE ALGORITMOS}

En esta sección se presenta una descripción de los tres algoritmos aplicados a la resolución del problema CVRP para la recolección de residuos tecnológicos. El algoritmo de ahorros y dos metaheurísticas Búsqueda Tabú y Optimización basada en colonia de Hormigas.

\subsection{Algoritmo de ahorros}

Este algoritmo es una heurística muy significativa para llevar a cabo la resolución de problemas de tipo VRP. Esta heurística es un procedimiento simple que realiza una exploración limitada del espacio de búsqueda y da una solución de calidad más o menos aceptable en tiempo de cálculo moderado. Si en una solución dos rutas diferentes $(0, \ldots, i, 0)$ $\mathrm{y}(0, j, \ldots, 0)$ pueden ser combinadas formando una nueva ruta $(0, \ldots, i, j, \ldots, 0)$. El ahorro (en distancia) obtenido por dicha unión es (ecuación 3):

$$
s_{i j}=c_{i 0}+c_{0 j}-c_{i j}
$$

En este algoritmo se comienza de una solución inicial y se realizan las uniones que den mayores ahorros siempre que no violen las restricciones del problema. 
Cuando el máximo ahorro es negativo, las combinaciones de las rutas aumentaran la distancia recorrida, pero disminuirá la cantidad de rutas de la solución, dependiendo de las características de cada problema esto puede generar rutas circulares o radiales que se pueden evitar colocando un valor referencial $\lambda$, el cual penaliza la unión rutas con clientes lejanos. Se propone el ahorro como:

$$
s_{i j}=c_{i 0}+c_{0 j}-\lambda c_{i j}
$$

A continuación, se presenta un pseudocódigo del algoritmo de ahorros:

Paso 1: Con las coordenadas de cada cliente o ciudad. Elaborar la matriz de distancias.

Paso 2: Calcular la tabla de ahorros $s_{i j}$ para cada par de clientes o ciudad $i, j=1,2, \ldots, n$.

Paso 3: Para cada cliente o ciudad $i$ construir la ruta $(0, i, 0)$.

Paso 4: Ordenar los ahorros de mayor a menor.

Paso 5: Iniciando por el mayor de los ahorros unir los nodos correspondientes, de tal forma que $s_{i j}=\max s_{i j}$, donde el máximo se toma entre los ahorros que no han sido considerados aún, se creará la ruta $r_{i * j *}$, si $i *$ es el último cliente de

$r i_{*}$ y $j *$ es el primer cliente de $r j *$. Eliminar $S_{i * j *}$ de futuras consideraciones. Se repite el paso 5 hasta que no haya más combinaciones de ahorros a considerar.

\subsection{Búsqueda Tabú}

La Búsqueda Tabú, es una estrategia de búsqueda propuesta originalmente por Glover (1989) adopta una estrategia específica para poder escapar de un mínimo local y continuar la búsqueda por otras soluciones posiblemente mejores.

En cada iteración el método realiza una exploración del espacio de soluciones moviéndose de una solución $S_{i}$ en la iteración $i$ a una solución vecina $S_{i+1}$ perteneciente al vecindario $N\left(S_{i}\right)$ de $S_{i}$, aun en caso de que $S_{i+1}$ sea peor que $S_{\mathrm{i}}$ logrando de esta forma escapar del mínimo local. Sin embargo, como justamente $S_{i+1}$ no necesariamente será una solución mejor se debe utilizar algún mecanismo para evitar ciclar sobre soluciones ya visitadas. Para éste $n$, la Búsqueda Tabú mantiene una estructura de memoria denominada lista tabú con el historial de soluciones visitadas o movimientos realizados en el pasado.

A continuación, se presentan los pasos más relevantes en la implementación del algoritmo de Búsqueda Tabú:

Paso 1: Generar una solución inicial, puede ser de manera aleatoria o a través de algoritmos heurísticos. Generar una solución de manera aleatoria puede traer grandes desventajas debido a que la solución encontrada puede ser de mala calidad; en el presente algoritmo las soluciones iniciales se generaron con la heurística del vecino más cercano.

Paso 2: Elegir el entorno o vecindario de la solución inicial, con el fin de generar nuevas soluciones a partir de la inicial. Para generar el vecindario se aplican técnicas como: Inserción, intercambio, etc. En este caso se utilizó una técnica de intercambio para generar el vecindario.

Paso 3: Evaluar la función objetivo. Si la solución es factible y mejor que la anterior se toma como nueva solución.

Paso 4: Se revisa que la nueva solución no esté restringida por un movimiento tabú, y se toma como nueva solución, si está restringida se toma la siguiente mejor solución factible y no restringida por la lista tabú. 
Paso 5: Se actualiza la lista tabú reemplazando el valor más antiguo de la lista por el nuevo movimiento tabú.

Paso 6: Se ejecuta el algoritmo hasta que se cumpla el criterio de parada. Este criterio puede ser definido por la cantidad de iteraciones, la cantidad de iteraciones sin mejora, el tiempo de ejecución, entre otros.

\subsection{Algoritmo ACO}

El algoritmo ACO fue propuesto por Dorigo et al. (1996) como metaheurística inspirada en el comportamiento de una colonia de hormigas en el proceso de forrajeo y aplicado principalmente para resolver problemas de optimización combinatoria. Este enfoque fue propuesto inicialmente cómo método para resolver el clásico problema del viajante de comercio (Traveling Salesman Problem).

En general en ACO, hay una colonia de $m$ hormigas (artificiales) que se mueven concurrente y asincrónicamente, a través de los estados adyacentes del problema. Se utiliza una regla de transición para realizar este movimiento, la que se basa en la información local disponible en los arcos del grafo que representa el problema (los nodos representan los puntos de recolección y los arcos los caminos que unen los puntos de recolección). Se utiliza una regla de transición para realizar este movimiento, la que se basa en la información local disponible en los arcos del grafo que representa el problema. Para guiar la búsqueda, esta información contiene tanto la información heurística $\left(\eta_{i j}\right)$ y los rastros de feromona $\left(\tau_{i i}\right)$ asociados a cada arco $(i, j)$. Las hormigas depositan feromona al transitar por un arco mientras construyen una solución (actualización en línea paso a paso de los rastros de feromona). Después que las $m$ hormigas generan una solución, se deposita una cantidad de feromona en los arcos de la mejor solución conocida (actualización en línea a posteriori de los rastros de feromona), de esta forma se guía la búsqueda de soluciones en el siguiente ciclo.

A continuación, se describen los pasos del algoritmo ACO:

Paso 1: Inicializar los parámetros de rastros de feromonas, factor de evaporación, número de hormigas y factores de información heurística.

Paso 2: Construir solución, cada hormiga debe decidir, a través de la regla de transición probabilística, ya sea para explorar nuevas conexiones o explotar las que ya han sido visitadas por otras hormigas.

Paso 3: Cada solución generada es una secuencia de nodos que ha sido visitado por una hormiga. Por tanto, las hormigas deben iniciar su recorrido desde algún nodo del grafo. Este nodo inicial se elige al azar para todas las hormigas en la colonia. El orden de las visitas de los nodos en camino depende de las decisiones del algoritmo

Paso 4: Inicializar los recorridos de las hormigas. Cada solución generada es una secuencia de nodos que ha sido visitado por una hormiga. Por tanto, las hormigas deben iniciar su recorrido desde algún nodo del grafo. Este nodo inicial se elige al azar para todas las hormigas en la colonia.

Paso 5: Cuando todas las hormigas terminan su recorrido, se ejecuta una rutina global (fuera de línea), que se encarga de actualizar los rastros de feromonas de hormigas que el algoritmo considera las mejores soluciones.

Paso 6: Si no se alcanzó el criterio de parada volver al Paso 2. 


\section{INSTANCIAS, EXPERIMENTOS Y RESULTADOS}

En esta sección se describen las instancias utilizadas, los experimentos realizados para resolver el problema y se analizan los resultados.

\subsection{Instancias generadas para la recolección de residuos tecnológicos}
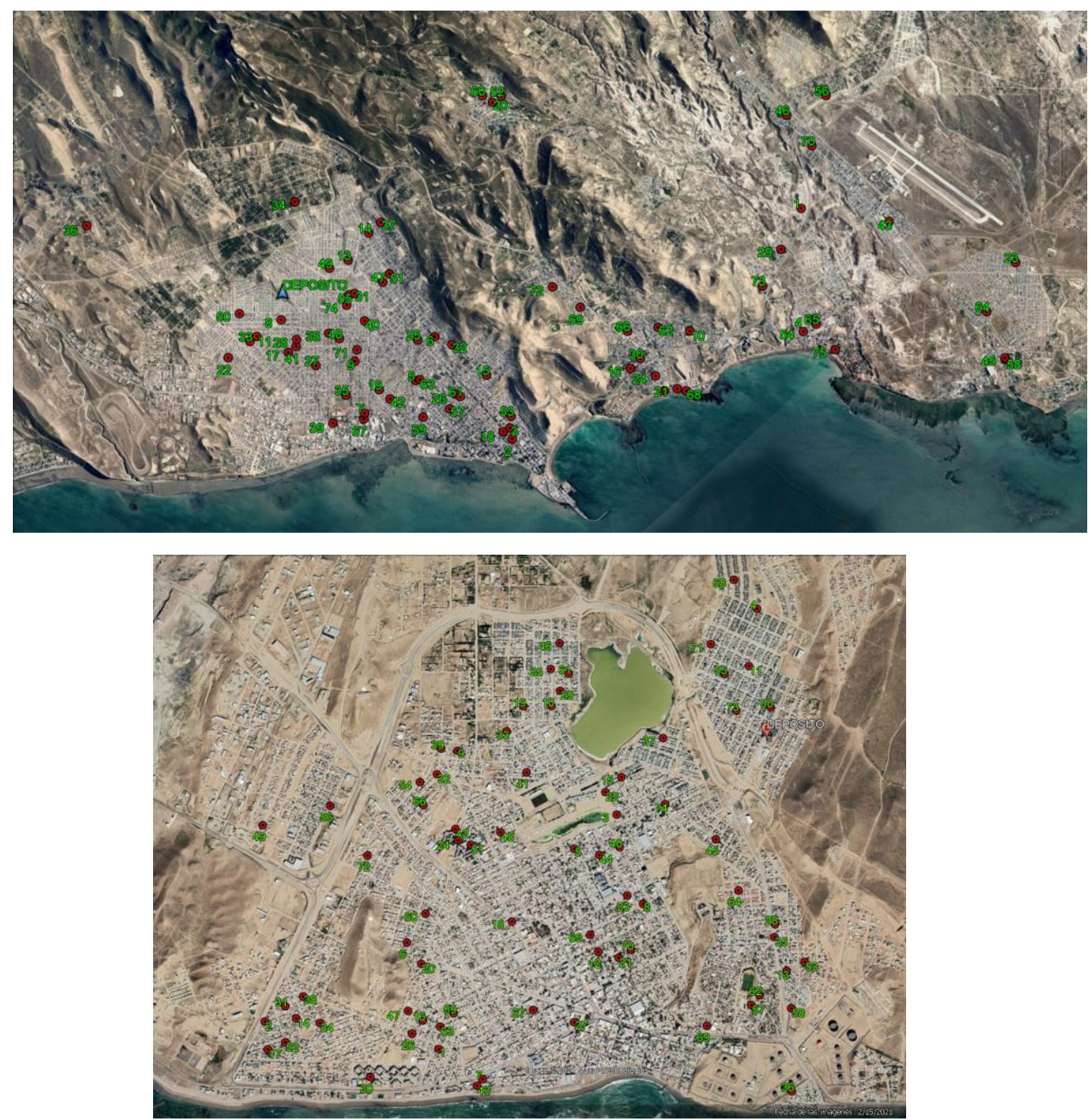

Figura 1. Distribución de 75 lugares de recolección en las ciudades de Comodoro Rivadavia (arriba) y Caleta Olivia (abajo)

Hemos generado dos instancias correspondientes a dos ciudades distintas, en las que se realizará la recolección de residuos tecnológicos.

La primera instancia corresponde a la ciudad de Caleta Olivia y la segunda instancia a la ciudad de Comodoro Rivadavia.

En ambas ciudades se realizará una recolección de residuos estacionaria y se definieron tres escenarios distintos de 75, 105 y 150 puntos de recolección de residuos tecnológicos, ubicados en lugares con disponibilidad para su gestión y cuatro escenarios para la ciudad de Comodoro Rivadavia con 75, 105, 150 y 200 puntos de recolección. En ambos casos los 
lugares corresponden a centros educativos o gubernamentales de cada una de las ciudades. En la Figura 1 se muestra la distribución de 75 lugares de recolección (en color rojo) y el numero identificador del lugar (en color verde) para la ciudad de Comodoro Rivadavia (imagen superior). Para la ciudad de Caleta Olivia se muestran también los 75 lugares de recolección (imagen inferior).

En cada uno de los puntos de recolección existen contenedores de igual tamaño con un volumen de $0,225 \mathrm{~m}^{3}$. Los vehículos utilizados para la recolección poseen capacidades distintas y tienen diferentes características. El primer vehículo denominado VH1 soporta una carga de hasta 1,5 toneladas y cubicaje de $16 \mathrm{~m}^{3}$, el costo promedio por $\mathrm{km}$ recorrido es de 1,38 U\$S, tiene un consumo de 0,30 litros de gasoil por $\mathrm{km}$ recorrido, tiene una capacidad de carga máxima de 71 contenedores y su carga y descarga es de tipo manual requiriendo 6 y 2 minutos respectivamente. De igual manera se detallan todos los vehículos en la Tabla 1.

Tabla 1: Vehículos de recolección de e-waste

\begin{tabular}{|l|l|l|l|l|l|l|}
\hline $\begin{array}{l}\text { Tipo de } \\
\text { Vehículo }\end{array}$ & $\begin{array}{l}\text { Peso de la } \\
\text { carga }\end{array}$ & $\begin{array}{l}\text { Cubicaje } \\
\text { Máximo } \\
\left(\mathbf{m}^{\mathbf{3}}\right)\end{array}$ & $\begin{array}{l}\text { Costo } \\
\mathbf{x ~ K m} \\
\mathbf{( U \$ S )}\end{array}$ & $\begin{array}{l}\text { Consumo } \\
\text { Medio } \\
\mathbf{( 1 / k m )}\end{array}$ & $\begin{array}{l}\text { Capacidad en } \\
\text { Contenedores }\end{array}$ & $\begin{array}{l}\text { Automatización } \\
\text { Carga y } \\
\text { Descarga }\end{array}$ \\
\hline VH1 & $\begin{array}{l}\text { Hasta 1,5 } \\
\text { Toneladas }\end{array}$ & $16 \mathrm{~m}^{3}$ & 1,38 & 0,30 & 71 & $\begin{array}{l}\text { Manual } \\
6 \text { min }-2 \text { min }\end{array}$ \\
\hline VH2 & $\begin{array}{l}\text { Hasta 2,5 } \\
\text { Toneladas }\end{array}$ & $22 \mathrm{~m}^{3}$ & 1,48 & 0,38 & 97 & $\begin{array}{l}\text { Semiautomático } \\
4 \text { min }-2 \text { min }\end{array}$ \\
\hline VH3 & $\begin{array}{l}\text { Hasta } 4.5 \\
\text { Toneladas }\end{array}$ & $32 \mathrm{~m}^{3}$ & 2,67 & 0,48 & 142 & $\begin{array}{l}\text { Automático } \\
4 \text { min }-1 \text { min }\end{array}$ \\
\hline
\end{tabular}

\subsection{Experimentos y resultados}

Los algoritmos seleccionados y la parametrización utilizada para el algoritmo de Búsqueda Tabú son de 200 iteraciones y para ACO la población se fijó en 50 hormigas y el máximo de iteraciones en 10.000, la evaporación global $p(r o)$ en 0,1 , la evaporación local ( $f i)$ en 0,1 y el valor inicial de feromona $T$ (tau) 0,0335. Los valores utilizados se han establecido en base a experimentaciones realizadas y de trabajos anteriores donde se han utilizado los algoritmos (Pérez et al., 2021).

Se realizaron 30 ejecuciones independientes de la Búsqueda Tabú y ACO aplicados a resolver el problema de recolección de e-waste.

Todos los algoritmos se implementaron en Java y se ejecutan con un procesador Intel i7 2.60 $\mathrm{GHz}$, con 8GB de RAM sobre Windows 10. En este trabajo, los mejores valores están marcados en negrita. En todos los experimentos se han analizado las condiciones que deben cumplirse para utilizar pruebas paramétricas y pruebas no paramétricas para el análisis estadístico con un error de probabilidad de $p=0,05$ utilizando RStudio Versión 1.1.463. Los mapas se han representado utilizando Google Earth Pro 7.3.4.8248.

Para el análisis de resultados se comparan los algoritmos propuestos para determinar si algunos de ellos muestran mejor calidad en los resultados (función de optimización) para cada uno de los escenarios propuestos. Para ello, se analizarán la mediana obtenida de las 30 
ejecuciones para los 9 escenarios propuestos que resultan de la combinación de 3 tipos de transportes diferentes y 3 (tres) cantidades distintas de contenedores, para la ciudad de Caleta Olivia. Para la ciudad de Comodoro Rivadavia se incorpora un escenario más que corresponde a 200 locaciones a recorrer y se mantienen los tres tipos de contenedores. Las

Tabla 2 y Tabla 4 muestran los resultados obtenidos por cada algoritmo.

$\mathrm{La}$

Tabla 2 muestra los resultados obtenidos por el algoritmo AA (una sola ejecución al tratarse de un método determinista) para los tres escenarios propuestos y la mediana de los kilómetros recorridos para 30 ejecuciones de los algoritmos TS y ACO en los tres escenarios propuestos. La columna de $N$ identifica cada escenario donde se indica la cantidad de locaciones a recorrer (75, 105 y 150). Los acrónimos C16, C22 y C32 representan la capacidad del transporte en metros cúbicos $\left(16,22,32 \mathrm{~m}^{3}\right)$. El cuadro central de la tabla detalla la cantidad de $\mathrm{km}$ recorridos por cada vehículo con distinta capacidad y escenario en cuanto a la cantidad de locaciones a visitar. Finalmente, en la última fila se observa los promedios de los $\mathrm{km}$ recorridos (no incluye al transporte al depósito final) obtenidos por cada algoritmo.

Tabla 2: Resultados de $\mathrm{Km}$ recorridos para los tres algoritmos con instancias de Caleta Olivia

\begin{tabular}{cccccccrrrr} 
& \multicolumn{3}{c}{ AA } & \multicolumn{3}{c}{ TS } & \multicolumn{3}{c}{ ACO } \\
\hline $\mathbf{N}$ & $\mathrm{C} 16$ & C22 & C32 & C16 & C22 & C32 & C16 & \multicolumn{1}{c}{ C22 } & \multicolumn{1}{c}{ C32 } \\
\hline $\mathbf{7 5}$ & 48,71 & $45, .67$ & 40,89 & $\mathbf{3 7 , 4 5}$ & $\mathbf{3 4 , 3 1}$ & $\mathbf{3 1 , 4 6}$ & 94,63 & 90,49 & 90,49 \\
$\mathbf{1 0 5}$ & 62,89 & 55,25 & 55,98 & $\mathbf{5 1 , 1 9}$ & $\mathbf{4 3 , 0 9}$ & $\mathbf{4 1 , 2 9}$ & 121,77 & 121,77 & 118,24 \\
$\mathbf{1 5 0}$ & 71,75 & 61,26 & 54,96 & $\mathbf{6 1 , 1 4}$ & $\mathbf{5 3 , 5 4}$ & $\mathbf{4 6 , 5 2}$ & 175,13 & 171,70 & 170,39 \\
\hline Prom & 61,12 & 54,06 & 50,61 & $\mathbf{4 9 , 9 3}$ & $\mathbf{4 3 , 6 5}$ & $\mathbf{3 9 , 7 6}$ & 130,51 & 127,99 & 126,37
\end{tabular}

En la Tabla 2 podemos observar que el algoritmo TS obtiene en mediana los menores valores en cuanto a $\mathrm{Km}$ recorridos para las tres escenarios para la ciudad de Caleta Olivia. Analizamos si los resultados obtenidos por estos algoritmos presentan diferencias estadísticamente significativas. Se verifica primero la normalidad (Shapiro-Wilk normality test), luego la homocedasticidad (Levene's Test for Homogeneity of Variance ) y atendiendo a los resultados obtenidos por estos test, se realizan test paramétricos o no paramétricos (T-test o U Mann-Whitney test) según corresponda.

En la Tabla 3 la columna $N$ identifica cada escenario donde indica la cantidad de locaciones a recorrer $(75,105$ y 150) para Caleta Olivia y la capacidad del transporte $(16,22,32 \mathrm{~m} 3)$. La columna Norm establece (con sí o no) si las muestras tienen una distribución normal través de aplicar el test de normalidad donde con 95\% de confianza se determina si alguna de submuestras de cada algoritmo son normales. La columna Hom establece (con sí o no) las muestras que poseen una distribución homogénea sobre la varianza a través de aplicar la prueba de homosedasticidad donde con $95 \%$ de confianza la mayoría de los resultados obtenidos no presenta homocedasticidad mientras que solamente dos presentan homocedasticidad. La columna T-Test / UMW corresponde a la aplicación de T-test cuando las columnas Norm y Hom son afirmativas y la aplicación de U Mann-Whitney test en caso contrario. Dados los resultados se puede decir con un 95\% de confianza que las diferencias entre los resultados obtenidos por TS y ACO son estadísticamente significativos. 
Se puede ver claramente estas diferencias en la Figura 2. Se han seleccionado tres instancias y se muestra una gráfica de box-plot donde se observa la marcada diferencia de las medianas obtenidas por TS con respecto a las obtenidas por ACO. También podemos observar la robustez de ambos algoritmos ya que hay poca variación en los resultados obtenidos en cada ejecución. Todos los resultados están alrededor de la mediana correspondiente a cada algoritmo.

Tabla 3. Análisis estadísticos de los resultados obtenidos por TS y ACO para Caleta Olivia

\begin{tabular}{cccc}
$\mathbf{N}$ & Norm & Hom & T-Test/UMW \\
\hline $75 \times 16$ & si & si & si \\
$75 \times 22$ & no & no & si \\
$75 \times 32$ & no & no & si \\
$105 \times 16$ & no & no & si \\
$105 \times 22$ & no & si & si \\
$105 \times 32$ & no & no & si \\
$150 \times 16$ & no & no & si \\
$150 \times 22$ & no & no & si \\
$150 \times 32$ & no & no & si \\
\hline
\end{tabular}
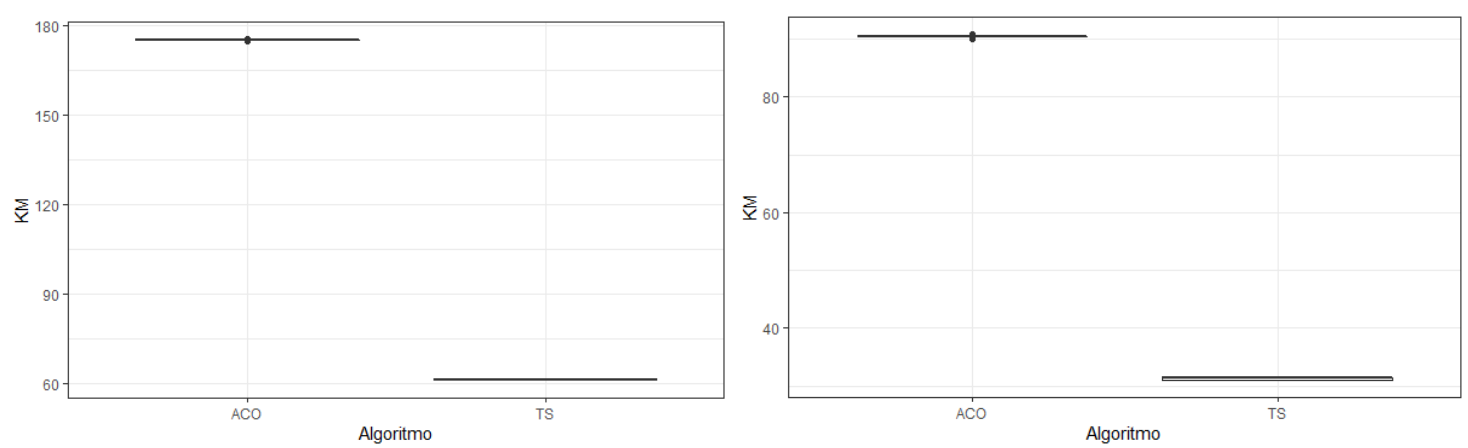

Figura 2. Box-plot de medianas de resultados para las instancias 75x32 (izquierda) y 150x32 (derecha)

La Tabla 4 muestra la misma información que la

Tabla 2, en este caso al tratarse de las instancias de Comodoro Rivadavia se tiene una fila más correspondiente a los 200 puntos de recolección que se definieron para esta ciudad.

Se puede observar que nuevamente para todas las instancias el algoritmo TS es el que obtiene los menores valores para los kilómetros recorridos.

Se realizan los análisis estadísticos correspondientes para determinar si las diferencias entre los resultados obtenidos por los algoritmos son estadísticamente significativas. La Tabla 5 muestra los resultados de la misma forma que se muestran en la Tabla 3 pero con la instancia de Comodoro Rivadavia. Nuevamente las diferencias de los resultados obtenidos por TS y ACO son estadísticamente significativos para todas las instancias de la ciudad de Comodoro Rivadavia.

Se eligieron dos resultados de dos instancias (75x22 y 200x32) para ver estás diferencias en los gráficos de box-plot que muestra la Figura 3. Podemos observar en la gráfica de la izquierda que algunos resultados de TS están por encima de su mediana no obstante hay una importante diferencia con respecto a los resultados obtenidos por ACO. En la gráfica de la derecha vemos que en general los resultados obtenidos por ambos algoritmos están alrededor de la mediana. 
Tabla 4: Resultados de Km recorridos para los tres algoritmos con instancias de Comodoro Rivadavia

\begin{tabular}{cccccccccc} 
& \multicolumn{3}{c}{ AA } & \multicolumn{1}{c}{ TS } & \multicolumn{3}{c}{ ACO } \\
\hline $\mathbf{N}$ & $\mathbf{C 1 6}$ & $\mathbf{C 2 2}$ & $\mathbf{C 3 2}$ & $\mathbf{C 1 6}$ & $\mathbf{C 2 2}$ & $\mathbf{C 3 2}$ & $\mathbf{C 1 6}$ & $\mathbf{C 2 2}$ & $\mathbf{C 3 2}$ \\
\hline $\mathbf{7 5}$ & 191,62 & 167,69 & 147,37 & $\mathbf{1 6 1 , 2 9}$ & $\mathbf{1 3 7 , 1 4}$ & $\mathbf{1 2 6 , 7 9}$ & 258,27 & 225,10 & 207,41 \\
$\mathbf{1 0 5}$ & 225,39 & 188,90 & 176,81 & $\mathbf{2 0 6 , 2 0}$ & $\mathbf{1 7 4 , 0 8}$ & $\mathbf{1 4 7 , 8 8}$ & 331,86 & 305,76 & 267,26 \\
$\mathbf{1 5 0}$ & 230,73 & 184,28 & 174,48 & $\mathbf{2 1 1 , 0 1}$ & $\mathbf{1 7 0 , 8 2}$ & $\mathbf{1 4 4 , 7 3}$ & 419,63 & 365,00 & 344,73 \\
$\mathbf{2 0 0}$ & 280,23 & 218,68 & 218,56 & $\mathbf{2 4 2 , 0 3}$ & $\mathbf{1 9 6 , 3 6}$ & $\mathbf{1 8 7 , 2 1}$ & 518,21 & 471,32 & 425,95 \\
\hline Prom & 231,99 & 189,89 & 179,31 & $\mathbf{2 0 5 , 1 3}$ & $\mathbf{1 6 9 , 6 0}$ & $\mathbf{1 5 1 , 6 5}$ & 381,99 & 341,79 & 311,34
\end{tabular}

Tabla 5: Análisis estadísticos de los resultados obtenidos por TS y ACO para Comodoro Rivadavia

\begin{tabular}{cccc}
$\mathbf{N}$ & Norm & Hom & T-Test/UMW \\
\hline $75 \times 16$ & no & no & si \\
$75 \times 22$ & no & no & si \\
$75 \times 32$ & no & no & si \\
$105 \times 16$ & no & no & si \\
$105 \times 22$ & si & no & si \\
$105 \times 32$ & no & si & si \\
$150 \times 16$ & no & no & si \\
$150 \times 22$ & no & no & si \\
$150 \times 32$ & si & no & si \\
$200 \times 16$ & no & no & si \\
$200 \times 22$ & si & no & si \\
$200 \times 32$ & no & no & si \\
\hline
\end{tabular}
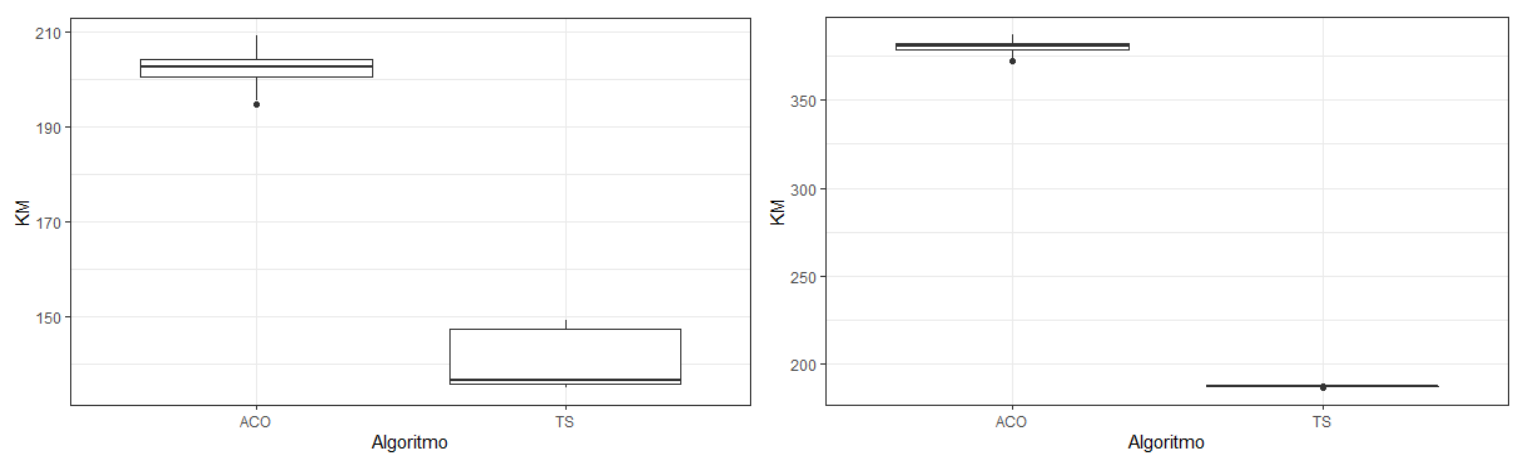

Figura 3. Box-plot de medianas de resultados para las instancias $75 \times 22$ (izquierda) y 200x32 (derecha)

En la Figura 4 y en la Figura 5 se muestran los mejores recorridos (menor $\mathrm{km}$ ) obtenidos por el algoritmo TS para una instancia de Caleta Olivia $(75 \times 16)$ y una instancia de Comodoro Rivadavia (75x16). En ambas figuras podemos observar en color rojo la ubicación del depósito y en color verde los distintos puntos de recolección. En la Figura 5 podemos observar que algunos puntos están muy alejados del depósito, esto se debe a la distribución de los diferentes barrios en la ciudad. 


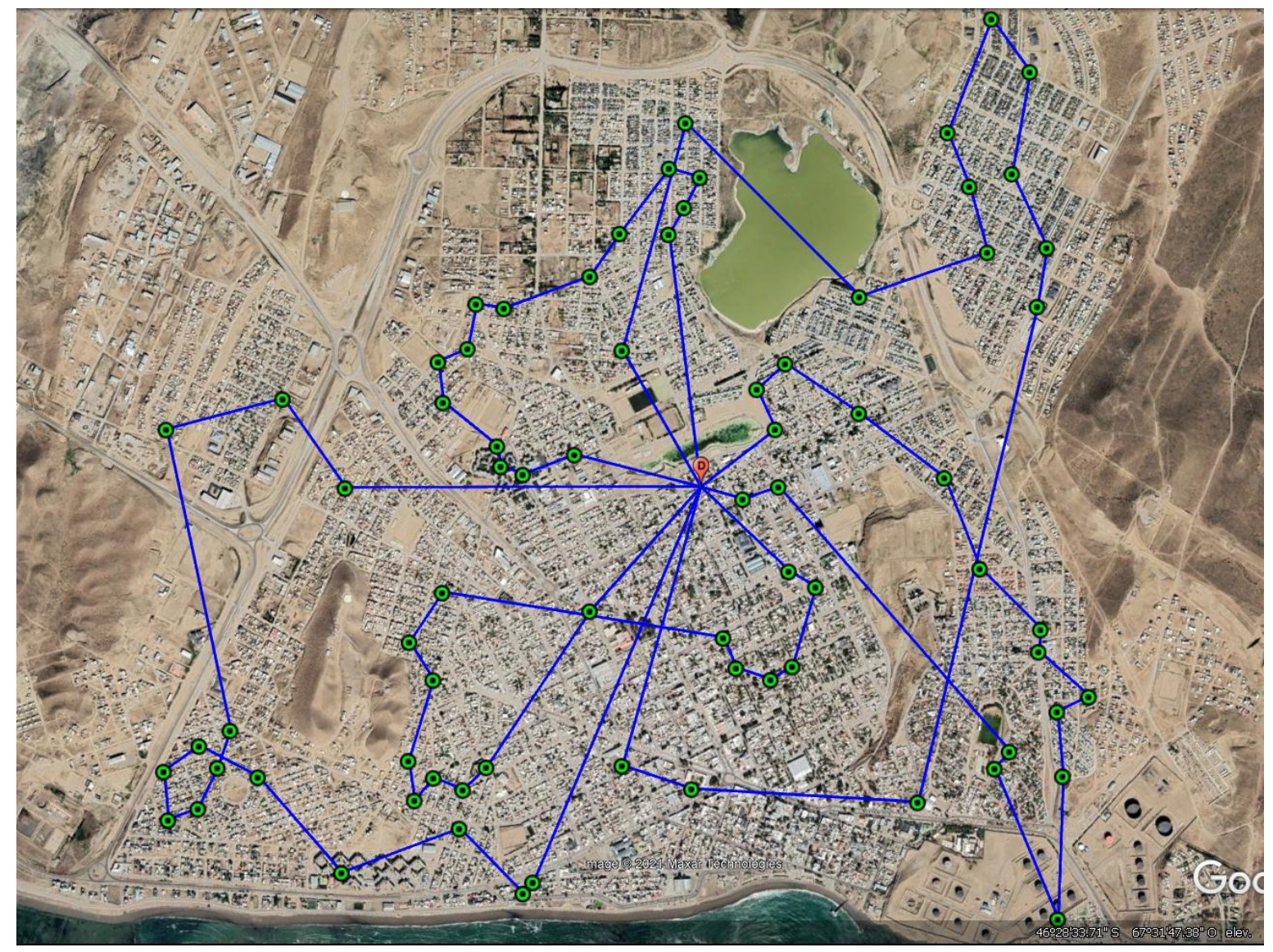

Figura 4: Mejor recorrido obtenido por TS para Caleta Olivia

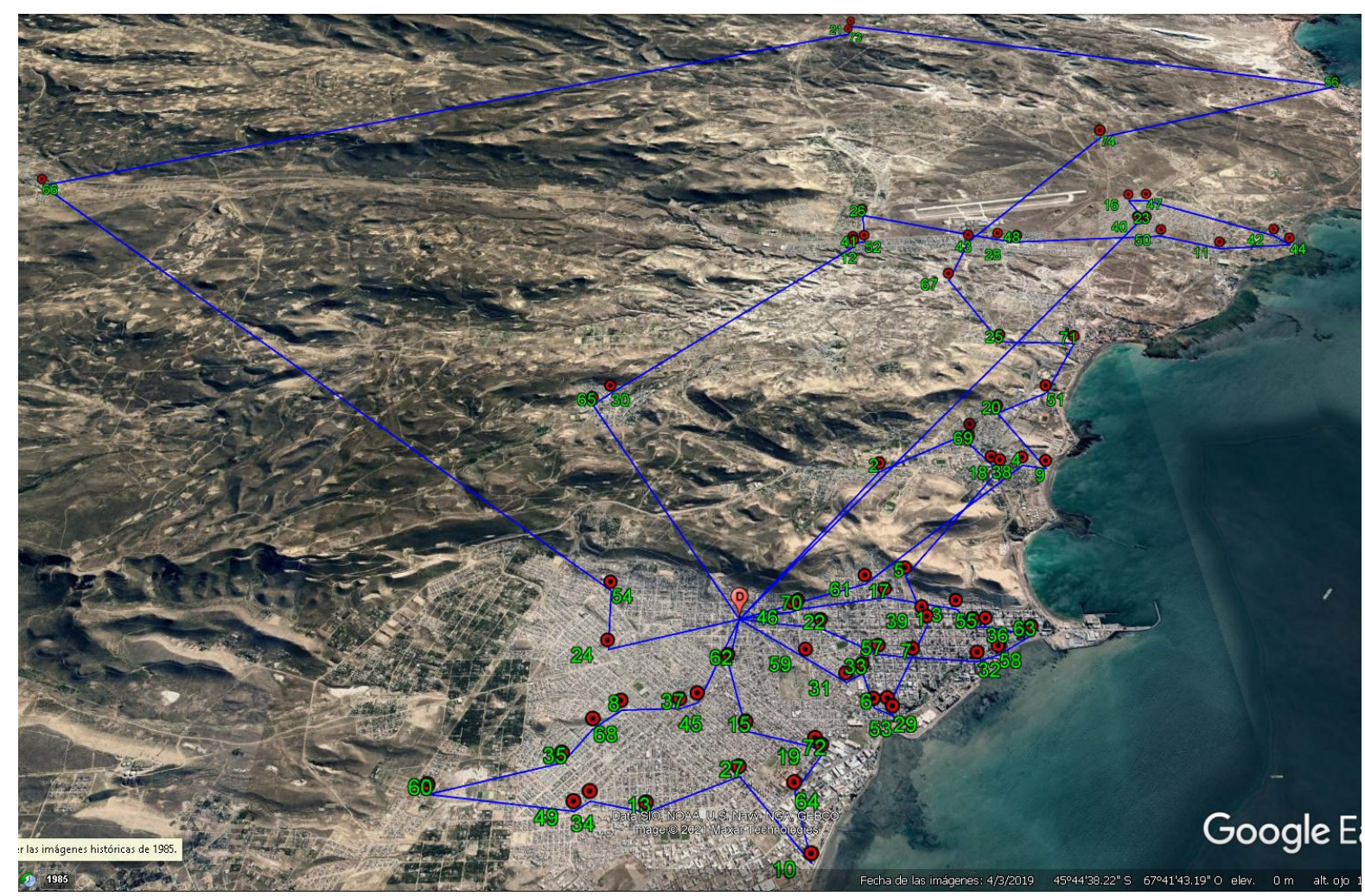

Figura 5: Mejor recorrido obtenido por TS para Comodoro Rivadavia 


\section{CONCLUSIONES}

El problema de recolección de residuos tecnológicos es un problema derivado del VRP cuyo objetivo principal es la minimización de los $\mathrm{km}$ recorridos para los distintos escenarios de recolección con locaciones predeterminadas y distintos tipos de vehículos.

Para resolver el problema se implementó una técnica heurística muy utilizada para este tipo de problema a la cual denominamos AA y dos técnicas metaheurísticas denominadas TS y ACO. Se aplicaron los algoritmos a escenarios reales de las ciudades de Caleta Olivia y de Comodoro Rivadavia. Se realizaron 30 ejecuciones independientes de TS y ACO, así como también los análisis estadísticos de los resultados obtenidos. El algoritmo TS es el que ha obtenido los menores valores para los kilómetros recorridos en cada una de las instancias con respecto a los otros dos algoritmos. Además, los resultados logrados por TS tienen diferencias estadísticamente significativas con respecto a los resultados obtenidos por ACO en todas las instancias. Con respecto a los resultados obtenidos por AA al tratarse se una heurística determinista arroja un solo resultado por cada instancia y se puede observar que estos resultados son menores a los obtenidos por ACO pero no menores a los obtenidos por TS. Finalmente, con un nivel de confianza del $95 \%$ podemos decir que TS para las instancias de Caleta Olivia y Comodoro Rivadavia obtiene los menores resultados de manera eficiente y robusta.

Como trabajo futuro se estudiará el comportamiento de este algoritmo (TS) y de otras metaheurísticas (en particular ACO) para instancias de mayor tamaño utilizadas en la literatura. Además, se realizará un análisis detallado de los gases efecto invernadero generados por los vehículos y la disminución de éstos al obtener recorridos óptimos.

\section{AGRADECIMIENTOS}

Los autores, agradecen al equipo de trabajo del Laboratorio de Tecnologías Emergentes (LabTEm) del Instituto de Tecnología Aplicada (ITA) y a la Universidad Nacional de la Patagonia Austral (UNPA), por la colaboración y por todo el financiamiento realizado en el desarrollo de este trabajo.

\section{REFERENCIAS}

ASIH, A. M. S., SOPHA, B. M., \& KRIPTANIADEWA, G. (2017). Comparison study of metaheuristics: Empirical application of delivery problems. International Journal of Engineering Business Management, 9, https://doi.org/10.1177/1847979017743603

BABAEE TIRKOLAEE, E., ABBASIAN, P., SOLTANI, M., \& GHAFFARIAN, S. A. (2019). Developing an applied algorithm for multi-trip vehicle routing problem with time windows in urban waste collection: A case study. Waste Management \& Research, 37(1_suppl), 4-13. https://doi.org/10.1177/0734242X18807001

BALARI, S. (2017). Desarrollo y complejidad computacional, ¿dos elementos clave para comprender los orígenes del lenguaje? Ludus Vitalis, 13(24), 181-198.

BERTERO, F. (2015). Optimización de recorridos en ciudades. Una aplicación al sistema de recolección de residuos sólidos urbanos en el Municipio de Concordia. Tesina de Grado. Universidad Nacional de Rosario. Rosario, Argentina. 
BIANCHETTI, M. L. (2015). Algoritmos de zonificación para recolección de residuos. Tesis de Grado. Universidad de Buenos Aires. Buenos Aires, Argentina.

BONOMO, F., DURÁN, G., LARUMBE, F., \& MARENCO, J. (2009). Optimización de la Recolección de Residuos en la Zona Sur de la Ciudad de Buenos Aires. Revista Ingenierı de Sistemas Volumen XXIII.

BONOMO, F., DURÁN, G., LARUMBE, F.; MARENCO, J. (2012). "A method for optimizing waste collection using mathematical programming: a Buenos Aires case study". Waste Management \& Research. Vol. 30, no. 3, págs. 311-324. https://doi.org/10.1177/0734242X11402870

BOUSSAÏD, I., LEPAGNOT, J., \& SIARRY, P. (2013). A survey on optimization metaheuristics. Information sciences, $237, \quad$ 82-117. https://doi.org/10.1016/j.ins.2013.02.041

CAVALLIN, A., VIGIER, H. P., \& FRUTOS, M. (2014). Logística inversa y ruteo en el sector de recolección informal de residuos sólidos urbanos. Avances en Gestión Integral de Residuos Sólidos Urbanos, 15, 37-49.

CLARKE, G., \& WRIGHT, J. W. (1964). Scheduling of vehicles from a central depot to a number of delivery points. Operations research, 12(4), 568-581. https://doi.org/10.1287/opre.12.4.568

DANTZIG, G. B., Y RAMSER, J. H. (1959). The truck dispatching problem. Management science, 6(1), 80-91. https://doi.org/10.1287/mnsc.6.1.80

DORIGO, M., MANIEZZO, V., \& COLORNI, A. (1996). Ant system: optimization by a colony of cooperating agents. IEEE Transactions on Systems, Man, and Cybernetics, Part B (Cybernetics), 26(1), 29-41. https://doi.org/10.1109/3477.484436

GHIANI, G., LAGANÀ, D., MANNI, E., \& TRIKI, C. (2012). Capacitated location of collection sites in an urban waste management system. Waste management, 32(7), 1291-1296. https://doi.org/10.1016/j.wasman.2012.02.009

GLOVER, F. (1986). Future paths for integer programming and links to artificial intelligence. Computers \& operations research, 13(5), 533-549. https://doi.org/10.1016/0305-0548(86)90048-1

GLOVER, F. (1989). Tabu search-part I. ORSA Journal on computing, 1(3), 190-206. https://doi.org/10.1287/ijoc.1.3.190

GU, F., ZHANG, W., GUO, J., \& HALL, P. (2019). Exploring “Internet+ Recycling": Mass balance and life cycle assessment of a waste management system associated with a mobile application. Science of the total environment, 649, 172-185. https://doi.org/10.1016/j.scitotenv.2018.08.298

LAPORTE, G., GENDREAU, M., POTVIN, J. Y., \& SEMET, F. (2000). Classical and modern heuristics for the vehicle routing problem. International transactions in operational research, 7(4- 5), 285-300. https://doi.org/10.1111/j.14753995.2000.tb00200.x

LEE, K. S., \& GEEM, Z. W. (2005). A new meta-heuristic algorithm for continuous engineering optimization: harmony search theory and practice. Computer methods in applied mechanics and engineering, 194(36-38), 3902-3933. https://doi.org/10.1016/j.cma.2004.09.007 
LENSTRA, J. K., \& KAN, A. R. (1981). Complexity of vehicle routing and scheduling problems. Networks, 11(2), 221-227. https://doi.org/10.1002/net.3230110211

MAR-ORTIZ, J., GONZÁLEZ-VELARDE, J. L., \& ADENSO-DÍAZ, B. (2013). Designing routes for WEEE collection: the vehicle routing problem with split loads and date windows. Journal of Heuristics, 19(2), 103-127. https://doi.org/10.1007/s10732-011$\underline{9159-1}$

NESMACHNOW, S., ROSSIT, D., \& TOUTOUH, J. (2018). Comparison of multiobjective evolutionary algorithms for prioritized urban waste collection in Montevideo, Uruguay. Electronic Notes in Discrete Mathematics, 69, 93-100. https://doi.org/10.1016/j.endm.2018.07.013

NOWAKOWSKI, P., KRÓL, A., \& MRÓWCZYŃSKA, B. (2017). Supporting mobile WEEE collection on demand: A method for multi-criteria vehicle routing, loading and cost optimisation. Waste Management, 69, 377-392. https://doi.org/10.1016/j.wasman.2017.07.045

NOWAKOWSKI, P., SZWARC, K., \& BORYCZKA, U. (2018). Vehicle route planning in e-waste mobile collection on demand supported by artificial intelligence algorithms. Transportation Research Part D: Transport and Environment, 63, 1-22. https://doi.org/10.1016/j.trd.2018.04.007

NOWAKOWSKI, P., SZWARC, K., \& BORYCZKA, U. (2020). Combining an artificial intelligence algorithm and a novel vehicle for sustainable e-waste collection. Science $\begin{array}{llll}\text { of The Total } & \text { Environment, } & 738726 .\end{array}$ https://doi.org/10.1016/j.scitotenv.2020.138726

PÉREZ, D., PANDOLFI, D., \& VILLAGRA, A. (2021). Recolección de Residuos Tecnológicos aplicando Metaheurísticas. Informes Científicos Técnicos-UNPA, 13(1), 54-76. https://doi.org/10.22305/ict-unpa.v13.n1.789

SUN, Q., WANG, C., ZHOU, Y., ZUO, L., \& TANG, J. (2020). Dominant platform capability, symbiotic strategy, and the construction of "Internet+ WEEE collection" business ecosystem: A comparative study of two typical cases in China. Journal of Cleaner Production, 254, 120074. https://doi.org/10.1016/j.jclepro.2020.120074

TOTH, P., \& VIGO, D. (Eds.). (2014). Vehicle routing: problems, methods, and applications. Society for Industrial and Applied Mathematics. https://doi.org/10.1137/1.9781611973594

YI, J., LU, C., \& LI, G. (2019). A literature review on latest developments of Harmony Search and its applications to intelligent manufacturing. Mathematical Biosciences and Engineering, 16(4), 2086-2117. https://doi.org/10.3934/mbe.2019102

ZANAKIS, S. H., \& EVANS, J. R. (1981). Heuristic “optimization”: Why, when, and how to use it. Interfaces, 11(5), 84-91. https://doi.org/10.1287/inte.11.5.84 